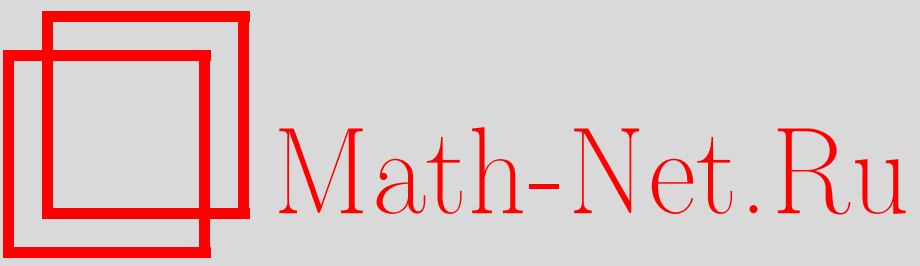

С. М. Гусейн-Заде, Ф. С. Дужин, О количестве топологических типов плоских кривых, УМН, 1998, том 53, выпуск 3, 197-198

DOI: https://doi.org/10.4213/rm48

Использование Общероссийского математического портала Math-Net.Ru подразумевает, что вы прочитали и согласны с пользовательским соглашением

http://www . mathnet.ru/rus/agreement

Параметры загрузки:

IP : 3.80 .181 .102

26 апреля 2023 г., $16: 56: 43$

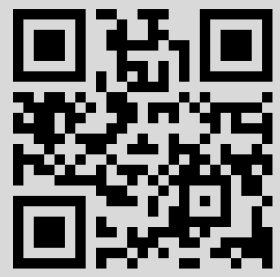




\title{
О КОЛИЧЕСТВЕ ТОПОЛОГИЧЕСКИХ ТИПОВ ПЛОСКИХ КРИВЫХ
}

\author{
С. М. ГУСЕЙН-ЗАДЕ,. С. ДУЖИН
}

Плоские кривые общего положения имеют в качестве особенностей только простые двойные самопересечения. Задача описания замкнутых кривых на плоскости и в пространстве (узлов) с точностью до гомеоморфизма плоскости или пространства восходит, по-видимому, к Гауссу. Обе задачи довольно сложны и не решены до сих пор. В [1] В. И. Арнольд высказал предположение, что "комбинаторика плоских кривых выглядит гораздо более сложной, чем теория узлов (которая может рассматриваться как упрощенная 'коммутативная' версия комбинаторики плоских кривых и которая, возможно, вкладьвается в теорию плоских кривых)". В [1] приведена статистика количества плоских кривых с не более чем пятью точками самопересечения. Перечисление кривых проводилось вручную и не было основано на каких-либо общих алгоритмах.

В [2] была рассмотрена задача классификации "длинных кривых" на плоскости. Длинная кривая - это образ общего невырожденного отображения прямой $\mathbb{R}$ в плоскость $\mathbb{R}^{2}$, совпадающего со стандартным вложением $x \mapsto(x, 0)$ вне некоторого ограниченного множества в $\mathbb{R}$. Был описан алгоритм перечисления этих кривых. Он основан на описании длинных кривых с помощью диаграмм специального вида, выделении подкласса диаграмм (так называемых нормальных), элементы которого находятся во взаимно-однозначном соответсвии с классами топологически эквивалентных длинных кривых, и алгоритме перечисления (без повторений либо проверки неподходящих вариантов) всех нормальных диаграмм. На основе этого алгоритма была разработана компьютерная программа, определяющая число длинных кривых с различным количеством точек самопересечения (см. последний столбец таблицы в конце заметки).

В настоящей работе вкратце описана модификация этого алгоритма, считающая число замкнутых кривых на плоскости.

Замкнутая плоская кривая (далее-просто кривая) - это образ гладкого невырожденного отображения окружности $S^{1}$ в плоскость $\mathbb{R}^{2}$ общего положения, т.е. имеющего в качестве особенностей толшко простые двойные самопересечения. Две кривые эквивалентны, если существует гомеоморфизм плоскости, переводящий одну из них в другую. При этом возникают четьре типа эквивалентности в зависимости от того, допускаются или нет гомеоморфизмы, меняющие ориентацию плоскости или окружности. Так, если допускаются толњко гомеоморфизмы, сохраняющие ориентацию плоскости, то можно говорить о классифокации кривых на ориентированной плоскости.

Длинной кривой соответствует замкнутая кривая, получающаяся соединением ее концов в верхней полуплоскости. (Различным длинным кривым может соответствовать одна и та же замкнутая.) При этом длинные кривые находятся во взаимно-однозначном соответствии с классами топологически эквивалентных неориентированных кривых на ориентированной плоскости с отмеченной точкой на одном из отрезков внешнего контура. Предположим, что замкнутая кривая, соответствующая данной длинной, получается из $m$ длинных кривых. Число $m$ равно количеству топологически неэквивалентных способов отметить точку на внешнем контуре замкнутой кривой. Количество неориентированных кривых на ориентированной плоскости равно сумме по всем длинным кривым величин $1 / m$. Величина $m$ для данной длинной кривой может быть определена следующим образом. Соответствующая точка на внешнем контуре замкнутой кривой последовательно смещается на один отрезок против часовой стрелки. Не представляет труда описать некоторую диаграмму соответствующей длинной кривой. Центральной частью алгоритма является процедура нормализации полученной диаграммы. Если нормализованная диаграмма совпадает с исходной, то эти длинные кривые эквивалентны. Длинная кривая, эквивалентная исходной, встречается после сдвига выбранной точки на $m$ отрезков.

Для вычисления количества длинных кривых относительно других типов эквивалентности (различающихся ориентированностью или неориентированностью плоскости или окружности)

Работа выполнена при финансовой поддержке Российского фонда фундаментальных исследований (грант № 98-01-00612). 
необходимо определить тип симметрии кривой ([1]). Пусть $\sigma: S^{1} \rightarrow S^{1}$ и $\Sigma: \mathbb{R}^{2} \rightarrow \mathbb{R}^{2}-$ стандартные отображения, меняющие ориентацию окружности и плоскости. Кривые бывают суперсимметричными: $[l]=[l \sigma]=[\Sigma l]=[\Sigma l \sigma],(1)$-симметричными: $[l]=[\Sigma l \sigma],(2)$-симметричными: $[l]=[l \sigma],(3)$-симметричными: $[l]=[\Sigma l]$, и асимметричными $([l]-$ класс ориентированных кривых на ориентированной плоскости, топологически эквивалентных кривой $l$ ). Эти кривые, рассматриваемые как неориентированные на неориентированной плоскости, вносят в четыре столбца таблицы 1 , соответствующие замкнутым кривым, вклады $(1,1,1,1),(2,1,1,1)$, $(2,2,1,1),(2,1,2,1)$ и $(4,2,2,1)$. Чтобы выяснить, например, является ли данная кривая (3)-симметричной, следует отразить диаграмму кривой относительно вертикальной оси (при этом получается, вообще говоря, не нормальная диаграмма), нормализовать ее и проверить, встречается ли полученная диаграмма среди нормальных диаграмм, соответствующих различным выборам точки на внешнем контуре рассматриваемой кривой.

Написанная на Паскале программа, определяющая количество замкнутых кривых различных типов, находится по URL-aдpecy http://www . botik.ru/ duzhin/dataprog/allcurve.pas

Расчеты дали резултаты, приведенные в следующей таблице:

\begin{tabular}{|c|c|c|c|c|c|}
\hline & \multicolumn{4}{|c|}{ Замкнутые кривые } & Длинные кривые \\
\hline ориентировано & $\mathbb{R}^{2}, S^{1}$ & $\mathbb{R}^{2}$ & $S^{1}$ & - & \\
\hline 0 & 2 & 1 & 1 & 1 & 1 \\
\hline 1 & 3 & 2 & 2 & 2 & 2 \\
\hline 2 & 10 & 5 & 5 & 5 & 8 \\
\hline 3 & 39 & 21 & 21 & 20 & 42 \\
\hline 4 & 204 & 102 & 102 & 82 & 260 \\
\hline 5 & 1262 & 639 & 640 & 435 & 1796 \\
\hline 6 & 8984 & 4492 & 4492 & 2645 & 13396 \\
\hline 7 & 67959 & 34032 & 34047 & 18489 & 105706 \\
\hline 8 & 544504 & 272252 & 272252 & 141326 & 870772 \\
\hline 9 & 4535030 & 2267905 & 2268085 & 1153052 & 7420836 \\
\hline 10 & 39004772 & 19502386 & 19502386 & 9819315 & 65004584 \\
\hline
\end{tabular}

\section{СПИСОК ЛИТЕРАТУРЫ}

[1] Arnold V.I. Topological Invariants of Plane Curves and Caustics. Providence: Amer. Math. Soc., 1994. [2] Gusein-Zade S. M. // Adv. Soviet Math. 1994. V. 21. P. 189-198.

Московский государственньй

Принято редколлегией университет им. М.В. Ломоносова

07.04.1998 